\title{
Putting the cart before the horse: co-evolution of the universe and observers as an explanatory hypothesis
}

Milan M. Ćirković ${ }^{1}$

Jelena Dimitrijević

\begin{abstract}
The answer to the fine-tuning problem of the universe has been traditionally sought in terms of either design or multiverse. In philosophy circles, this is sometimes expanded by adding the option of explanatory nihilism - the claim that there is no explanation for statements of that high level of generality: fine-tunings are brute facts. In this paper, we consider the fourth option which, at least in principle, is available to us: co-evolution of the universe and obsevers. Although conceptual roots of this approach could be found already in ancient stoicism, it is still the least investigated explanatory option for resolving the problem of empirical fine tunings. We offer two preliminary models along which the co-evolution hypothesis could be developed further. They are still on the level of speculative metaphysics, but there are opportunities along the way to generate predictions which are in principle testable, especially in the domain of large-scale numerical simulations.
\end{abstract}

Keywords: philosophy of cosmology - fine tuning - anthropic principles - practical metaphysics - numerical simulations - philosophy of biology - endosymbiosis - closed timelike curves - causation

The next great era of awakening of human intellect may well produce a method of understanding the qualitative content of equations. Today we cannot. Today we cannot see that the water flow equations contain such things as the barber pole structure of turbulence that one sees between rotating cylinders. Today we cannot see whether Schrödinger's equation contains frogs, musical composers, or morality-or whether it does not. We cannot say whether something beyond it like God is needed, or not. And so we can all hold strong opinions either way.

Richard Feynman, Lectures on Physics II, 41-12

\section{Introduction: the problem of fine-tunings apart from the design and the multiverse}

It is an empirical fact that physical constants and cosmological parameters of our universe are finetuned to enable habitability, abiogenesis, and emergence of observers like us. ${ }^{3}$ In contrast to

\footnotetext{
${ }^{1}$ Astronomical Observatory of Belgrade, Belgrade 11000, Serbia \& Future of Humanity Institute, Oxford University, Oxford, UK; e-mail: mcirkovic@aob.rs.

${ }^{2}$ Faculty of Philosophy, University of Belgrade; e-mail: idjecadimitrijevic@gmail.com.
} 
numerous mystifications, obfuscations and confusions, this epistemic status of fine-tunings is entirely uncontroversial: fine tunings are empirical facts. A small change in several of the dimensionless fundamental physical constants or cosmological parameters would make the universe radically different and uninhabitable; empirically, we are here, hence the universe is in fact habitable, hence those constants and parameters are confined to small intervals of values, much smaller - even infinitely smaller in some cases - than the interval in which they could a priori be. For instance, "a change of more than $0.5 \%$ in the strength of the strong interaction or more than $4 \%$ change in the strength of the Coulomb force would destroy either nearly all C or all O in every star" (Oberhummer, Csoto, and Schlattl 2000, p. 90). Since all forms of life we know so far depend crucially on both carbon and oxygen been created in previous generations of stars, the universe with the coupling constants for strong or electromagnetic interaction off by a few percent would not be habitable. And yet, there is no reason known to science thus far why these couplings could not, in principle, be different by million percent from their observed values.

What is controversial, however, is (i) whether there is an explanation of fine-tunings, (ii) whether it is worth searching for such an explanation, and (iii) if the answer to (i) and (ii) is affirmative, what is the best explanatory hypothesis for the explanandum of fine-tunings. Usually one starts from the outcome, namely from two famous explanatory hypotheses - or, more precisely, two classes of hypotheses - the Design Hypothesis and the Multiverse Hypothesis. ${ }^{4}$ These are the two most elaborate explanatory paradigms for fine-tunings. Either the observable universe was designed by natural or supernatural agency to be habitable and friendly to observers - or it is just a minuscule part of a much larger whole, the multiverse, which is consistent with all pre-requisites for our existence as observers.

It is worth a moment, however, to backtrack a bit and ask about further explanatory possibilities. Scientists and philosophers skeptical (for various and often conflicting reasons) about extravagant metaphysical claims of both Design and Multiverse hypotheses occassionally reject the idea that statements of such generality as those expressing values of fundamental physical constants and cosmological parameters have causal explanation in the first place. Instead, we shall hear from time to time, they are brute facts. Consequently, searching for their explanation - and other aspects of the initial conditions of the universe - is fruitless waste of time and energy (e.g., Callender 2004; Mosterín 2005). Still more sophisticated sub-branch of this view argues that the request for explanation is illegitimate, since there is no well-defined explanandum (Manson 2000; McGrew, McGrew, \& Vestrup 2001). This explanatory nihilism, provoked partly no doubt by the extravagancy of both Design and Multiverse, represents perhaps the "silent majority" view among scientists and philosophers. It seems common-sensical to acknowledge that there are questions "too big to answer", or at least too big from the point of view of cost-benefit analysis.

(From time to time, explanatory nihilism is interpreted as saying that the universe is habitable through dumb luck, like a coin landing heads a hundred times in a row. We know that there are occurences in everyday life - and occasionally in science as well - which occur due to dumb luck;

\footnotetext{
${ }^{3}$ For some of the reviews of already voluminous literature on this topic, see Carr and Rees (1979); Barrow \& Tipler (1986); Hogan (2000); Davies (2006); Barnes (2012).

${ }^{4}$ In further text, we shall treat each of them as single hypotheses, as denoted by capital letters, while fully admitting that these are just placeholders for their respective categories or families of hypotheses.
} 
serendipitous discoveries such as radioactivity, penicillin, or the cosmic microwave background are sometimes described in this manner. In the cosmological case, however, this is simply inapplicable, for the following reason. Consecutive coin tosses, whose outcomes constitute what we would describe as lucky accident, need to be real, physical events, not abstract possibilities; in the cosmological case, we would need multiple instantiations of "the universe" - which would then lead us to the multiverse hypothesis, rather than to the explanatory nihilism. It is of no consequence whether multiple universes are co-existing in some higher-dimensional superstructure or they for a temporal sequence, as in the old scenario of oscillating universe. Observational selection within a multiverse would in any such case take care of the fine tuning, and we would need to worry "only" about the physical mechanism for generating the ensemble of universes.)

More serious consideration shows, however, conceptual and practical bankruptcy of explanatory nihilism. For starters, the history of science clearly shows that, for instance, the question of whether Sun revolves around the Earth or vice versa was for very long time also considered "too big" or even "metaphysical" question which mortals could not hope to ever answer. So, for example, great medieval Islamic astronomers like Abu Rayhan Al-Biruni and Nasir al-Din Tusi were clearly aware of both geocentric and heliocentric systems, but expressed opinion that the issue is too complex to be ever entirely settled. Even more, delving into such matters was once considered pretentious, impertinent, or even sacrilegious. There are many other historical instances in which similar ignorabimus has eventually been shown to be unnecessary and unfounded.

This same debate has been led more than once in the history of physical cosmology, especially during the "Great Controversy" between the Big Bang and the steady-state theories of the expanding universe in the 1948-1965 period (Kragh 1996). As the distinguished cosmologist William Bonnor wrote: ${ }^{5}$

It is the business of science to offer rational explanations for all the events in the real world, and any scientist who calls on God to explain something is falling down on his job. This applies as much to the start of the expansion as to any other event. If the explanation is not forthcoming at once, the scientist must suspend judgment: but if he is worth his salt he will always maintain that a rational explanation will essentially be found.

Further, explanatory nihilism is irritable due to attempts to assign an aura of "deep" philosophical sophistication to our ignorance. Neither Callender nor Mosterin nor any other supporter of explanatory nihilism would suggest that the question Why does the stairway in your house consist of exactly 16 steps? requires answering in terms of brute facts. It is likely that they would quote causes connected with principles of masonry, efficiency, standards, height of an average person, etc. There is no "suspension of judgement" on such issues. However, to answer the question Why is the level of primary fluctuations in the cosmic microwave background $10^{-5}$ ? (cf., Tegmark \& Rees 1998) it is tempting to invoke brute facts, suspension of judgement, and impossibility of causal explanation. Is it only because we tacitly assume to know more about masonry than about cosmology?

Alleged simplicity of explanatory nihilism is indeed quite misleading, since its proponents do not list the statements claiming to be irreducible and inexplicable brute facts. But such a list must exist, by

\footnotetext{
${ }^{5}$ Bonnor (1964), p. 119.
} 
definition, if explanatory nihilism is to be a coherent doctrine. We can assume that the list contains statements like, for example, the following:

$\mathrm{P}_{\mathrm{k}}$ : Initial conditions at the Big Bang were such that the dark energy cosmological density fraction $\Omega_{\lambda}$ today is neither less than -5 nor greater than $5 .{ }^{6}$

This statement cannot, on the present level of understanding of fundamental physics and cosmology, be explained by any other statement or a set of statements. The same, however, can be said about the following statement:

$\mathrm{P}_{\mathrm{m}}$ : Initial conditions at Earth's crust formation 4,556 million years before present were such that the number of continents of Earth today is neither less than 2 nor greater than 18.

Why this statement is not a brute fact? Are $P_{k}$ and $P_{m}$ really that different? If yes, what exactly makes them different? Are proponents of explanatory nihilism ready to recommend to geo-scientists to treat statements like $P_{m}$ as brute facts? And are they ready to do so with the same nonchalance they apply to cosmologists in the case of statements like $P_{k}$ ? Finally, if the answer to the last two questions are affirmative, does that mean that we need to deal with large number of brute facts, surfacing in almost any scientific discipline? In our view, this is almost a reductio of the nihilist doctrine: many brute facts mean, rather, that we are too lazy to dig for explanations deep enough.

Finally, explanatory nihilism has one essential methodological demerit - which some proponents seemingly deem a merit instead (in particular, see Mosterín 2005) - namely that we can always return to it, if other directions of research and other explanatory schemes fail. This a posteriori adaptiveness has long ago (about the time of Democritus' and Epicurus' atomism) stopped being a virtue and is now more a burden for its proponents.

On the other hand, if we renounce explanatory nihilism (at least for a time being, while exploring other options), is it necessary to accept the Design or the Multiverse? The answer is no, since we have a fourth option, which is what we dub coevolution of universe and observers. ${ }^{7}$ It is much less developed and discussed than the rest of the field, but there are multiple reasons why those dissatisfied with both Design and the Multiverse might wish to investigate it. The core idea of coevolution is based upon an extrapolation of process metaphysics: if we perceive a correlation between the two outcomes (in this case the large-scale properties of the universe and our properties as observers) we should investigate whether there are correlations in processes leading to those outcomes (cosmological evolution vs. biological evolution and noogenesis). It is only reasonable to expect hidden correlations between parameters describing/governing these processes. In the most extreme version, we could ask whether it is one and the same underlying process. ${ }^{8}$ In this sense we

\footnotetext{
${ }^{6}$ This is certainly a true statement as pertaining to the observable universe at present epoch. (Of course, we may go further and claim that $\Omega_{\lambda}$ is, in fact, equal to $0.71 \pm 0.01$; e.g., Hinshaw et al. [2013].) The point, however, is something else: counterfactual or parallel universes in which dark energy density is outside these bounds almost certainly fail to evolve observers.

${ }^{7}$ Somewhat wider background for all four approaches to explanation of fine tunings is given in Ćirković (2012). See also Davies (2007) for an incisive criticism of all approaches, although Davies somewhat underestimates the strength of explanatory nihilism, at least among philosophers.

${ }^{8}$ One should note that such an approach looks much less eccentric if we keep in mind that within process metaphysics it is processes which are irreducible, primitive concepts, not material entities or events.
} 
use the locution "coevolution": two correlated processes which involve strong and repeated interactions or, alternatively, a single fundamental process which has two manifestation within a well-defined domain of space, time, and energy. It is not as if we could assert causal primacy of one process over another, no matter how close our biased viewpoint were to one of them.

Obviously, this understanding of coevolution is sufficiently similar to the strict definition of coevolution within classical biology: reciprocal evolutionary change in the interacting species (e.g., Thompson 1994). The main difference lies in historical fact that in the practice of biology (and most of the philosophy of biology) species are the central entities, whose characters present relevant explanandums. If we abandon species-centric perspective in favor of the perspective emphasizing the process and mechanisms of evolution - which bring about observed species and their specific characters as current and temporary outcomes - we shall obtain a similar coevolutionary concept.

Among a small number of thinkers who entertained coevolutionary thinking in the context of fundamental physics and cosmology, the key role belongs to the great physicist, philosopher, and science teacher John Archibald Wheeler. Wheeler's version of the co-evolution hypothesis was based on a specific subjectivist reading of the orthodox Copenhagen interpretation of quantum mechanics. On this reading, collapse of wavefunction occurs only through interaction with an observer (Wheeler 1975, 1977; Stapp 2007). Remembering Bohr's insistence on separation between micro- and macrolevel of description, the question what exactly is necessary for macroscopic description to be valid is entirely legitimate. If the answer contains (at least) an observer, it follows that emergence of the macroscopis description cannot be separated from the issue of evolution of observers in general. In other words, we cannot limit ourselves to a narrow class of quantum experiments (Stern-Gerlach, spectroscopic data, electron diffraction, and such things to which the quantum measurement problem is conventionally applied), but to much wider physical and biological reality around us. We have to involve the evolutionary background of observers, including for instance presence and properties of the Earth, the Solar System, the Milky Way, and everything else we usually think of as prerequisites for the emergence of life and intelligence (on Earth). Therefore, according to Wheeler's conception of the participatory anthropic principle, instead of a causal series, we deal with a feedback loop which links concrete physical reality and observers. Concrete physical reality here means the collapsed wavefunction, in contrast to the unitary evolving superposition of states. Instead of the universal, non-collapsed network of all physically possible outcomes, including both dead and alive Schrödinger's cat, the interaction with an observer (which we conventionally dub "the measurement") produces concrete individual outcomes constituting the classical world we see around us. ${ }^{9}$ Since the world around us is, as far as we can observe, a collection of concrete outcomes and not quantum superpositions, Wheeler found it a sufficient justification for the following general statement.

Participatory anthropic principle: Observers are necessary to bring universe into being.

Conventionally understood entities are just outcomes or, in a more general case, phases of different processes. For a modern introduction into process metaphysics see, for instance, Rescher (2000).

${ }^{9}$ It could be argued as well (vide Everett) that this measurement of live or dead cat impacts the observer herself, i.e., represents continuation of her evolution in the world. 
In Wheeler's other words: ${ }^{10}$

Quantum mechanics has led us to take seriously and explore the directly opposite [to classical reductionism] view that the observer is as essential to the creation of the universe as the universe is to the creation of the observer.

Note that this has prima facie nothing to do with fine tunings of the universe. In order to establish the link, we need an implicit assumption of self-consistency: the universe being brought into being is compatible with the particular sort of observers which are bringing it into being. While this may seem obvious, it does raise interesting questions like: what different sorts of observers are there? Can different sorts of observers be meaningfully said to inhabit the same universe? etc. We do not intend to argue that these questions necessarily present difficulties for the Wheelerian project; further careful philosophical analysis would be required for that.

Wheeler's approach through the participatory anthropic principle is not, however, the only way to elaborate and model co-evolutionary hypothesis for explanation of empirical fine tunings. In a general metaphysical context, other versions are conceivable, two of which will be outlined in the rest of this paper. They are intentionally formulated in such a way to be both syntaxically and semantically in contrast to the prevailing narratives of modern philosophy of physics and cosmology. In reflecting upon them, we are guided by two general themes. The first theme is our Popperian contention that provocation is a promising path to critical insights. The second theme is well-known historical scenario: a naive metaphysical speculation leading to more precise, and ultimately empirically falsifiable predictions of physical theory; it happened quite a few times on the record.

\section{Coevolution hypothesis: an endosymbiotic model}

In which manner could the universe and cognitive processes within it coevolve? An attempt to answer this question includes exploring of other examples of coevolution known from our empirical databases and drawing some analogies. Each such example is, of course, part of the physical universe - so that, if we accept coevolution as the explanation for fine tuning, they would be instances of double coevolution; of course, this does not influence their heuristic value.

The most obvious example in this sense is the endosymbiotic theory of the origin of eukaryotic cell in evolutionary biology and microbiology. From the early days of evolutionary theory, and especially since the establishment of the Tree of Life containing both prokaryotes and eukaryotes, the puzzle of the huge structural difference between a prokaryote cell - for instance, a bacterium - and an eukaryote cell - for instance, a neuron or a trombocite - was looming large. A bacterial cell is small and diffuse, while in the eukaryote cell we find multiple levels of organelle structure. Organelles, such as nuclei, vacuoles, mitochondria, possess complex internal structure and are exchanging energy and information with their environment through their membranes. Obviously, prokaryote cells originated first - and they have been extremely well-adapted to practically all terrestrial environments throughout the history of life (e.g., Gould 1996). Such superb bacterial adaptation immediately makes the question about the origin of eukaryotes much more difficult. In the

\footnotetext{
${ }^{10}$ Wheeler (1977), p. 27.
} 
memorable words of Isidore Rabi, who ordered that? What kind of selection pressure might have caused such a quantum leap in complexity - and fragility?

For quite a long time, there was no coherent idea about that - to a large degree due to the fact that the boundary is so sharp and there are no transitional forms between prokaryotes and eukaryotes. It is a true discrete step, one of the "major transitions" in evolution (Maynard Smith \& Szathmary 1997). To explain it, a revolutionary change in our thinking about the very concepts was required the approach which is the endosymbiotic theory. In its modern form, it has been formulated in the seminal study of Lynn Margulis (then Sagan) in 1967. ${ }^{11}$ In a simplified form, the endosymbiotic theory suggests that prokaryotes which for a very long time lived in the same ecosystem first developed symbiosis, and subsequently began to live one within another for reasons of efficiency of resource processing and metabolism. In the evolutionary deep time, one symbiont became the eukaryiotic cell, and others became its organelles. This opened a new, previously inaccessible part of the overall biological morphospace for further evolution - the huge biological complexity we observe today in Earth's biosphere is a consequence of that simple "trickery" of endosymbiosis. The endosymbiotic theory not only explains the origin of organelles within the eukaryotic cell, but also some aspects of their structure (internal membranes, for instance) and their functional specialization. Its most famous prediction, the existence of relict DNA within present-day organelles like mitochondria or citoplasts as characteristic "fossil" of the epoch of their separate living, has been triumphantly confirmed many decades after the original study of Margulis (e.g., McFadden 2001; Kutschera \& Niklas 2005).

Can the universe look like that? More precisely, can investigation of all possible functional dependencies lead to the solution of the problem of arising complexity through the emergence of observers? As long as we stay in the firm embrace of mechanistic reductionism, such a thing sounds nonsensical - at best - just as the proverbial putting the cart before the horse. It is an expression of our prejudices, however, since there is in fact nothing inherently bad in putting the cart before the horse as long as we don't have additional insights into wider context: dynamics of motion of carts and horses, requirements of anatomy and of traffic. One should mention that during the late 19th and early 20th century there were multiple experiments, especially in France, with carts which indeed were placed in front of the horse, i.e., pushed by horses. Thus, for instance, Windsor (1907):

The very latest Paris novelty in the vehicle line is a four-wheeled surrey in which the cart is actually before the horse... This 1-hp motor starts and stops on command, and has two speeds forward; the machine is not constructed to reverse. No lines are used, the conveyance being directed by means of a steering wheel. The outfit has not yet been arrested for fast driving.

Although such contraptions never became widely accepted or popular, that contingent fact of history only proved their impracticality, not that they were logically impossible, nor indeed technically unfeasible. An analogous argument should be, on the present level of cosmological understanding, sufficient for establishing legitimacy of coevolutionary approach to explanation of empirical fine tunings. We need to investigate many specific details of the hypothesis before concluding that it is impossible or unfeasible.

\footnotetext{
${ }^{11}$ Sagan (1967); this paper has been rejected about 15 times in different journals prior to publication.
} 
Therefore, significant amount of additional information is necessary before we decide whether such a radical hypothesis is indeed in conflict with scientific methodology - or only in conflict with our naive and intuitive prejudices how explanatory hypotheses should look like. It is uncontroversial that, due to our inability to sample outside of our past light cone (nor indeed in many regions of our past light cone due to physical constraints), we cannot observe the whole of the hypothetical cosmological endosymbiosis. Instead, we are facing a series of partial insights into the underlying evolutionary processes and partial insight often leads into difficulties when the underlying functional importance needs to be assessed. In evolutionary biology, the usual task is to reconstruct phylogenetic origin of a character on the basis of its functional properties; here we face more difficult forensic issue - how to reconstruct the underlying process on the basis of chunks and bits of empirical data on the universe as well as on the cognitive system, although those chunks and bits in themselves do not carry functional importance. For example, we observe Lyman- $\alpha$ absorption line systems in spectra of distant quasi-stellar objects (QSOs), but apart from very general properties of our perception required, there is no discernible relationship between their properties and properties of human cognition. The latter relationship, however, could be entirely sensible in the context of process metaphysics and the coevolutionary hypotheses, since by observing distant QSOs we observe distant past of the universe in which there were fewer (if any) observers and the cognition was certainly rarer and weaker in both quantitative and qualitative terms. Therefore, the "requirements" for fine tunings might have been weaker.

There is an ancient organicist tradition which have had some similar aspects to the endosymbiotic model. It could be found in stoicism, finely put for instance by Marcus Aurelius: ${ }^{12}$

Constantly regard the universe as one living being, having one substance and one soul; and observe how all things have reference to one perception, the perception of this one living being; and how all things act with one movement; and how all things are the cooperating causes of all things which exist; observe too the continuous spinning of the thread and the contexture of the web.

While we think about those symbiots becoming "mere" organelles in the eukaryotic cell as losing their identity in the process, this is not necessarily so and it hints more to the limits of the biological analogy than to additional insight into the ideas of cosmological coevolution. Instead, we can follow the stoics (and many subsequent organicists) into allowing the constituents to not only retain, but strengthen their concrete identities in the course of the evolutionary process.

Predictions which could be drawn from this coevolutionary model might be as follows. We need finer grading - or a continuum - of cognition within "non-living" as well as the "living" matter; this is in agreement with the "extended mind" ideas within recent philosophy of mind). We should expect algorithmic complexity, and in particular information capacity, to increase with passage of cosmic time in both living and non-living systems. This would be analogous to the prediction of the mitochondrial DNA in the original endosymbiotic theory.

\footnotetext{
${ }^{12}$ Marcus Aurelius, Meditations, book 4, translated by George Long (available online at http://classics.mit.edu//Antoninus/meditations.html, last accessed August 15, 2016).
} 


\section{Coevolutionary hypothesis: closed timelike curves}

Alternatively, let us try to imagine some of the future achievements of advanced cognition, in a distant epoch in which a large fraction - or perhaps most - of the physical universe is under control of intelligent species. If our experience so far tells us that the last part of the history of the universe is characterized by expansion of the influence of advanced cognition on its biological and physical environment, then we are entitled to ask: what could be long-term consequences of such a trend continuing? One of the possibilities is the use of closed timelike curves to influence the "past"13 and the universe made even more amenable to life and intelligence. Since Kurt Gödel first showed that closed timelike curves are, under particular conditions, acceptable within general relativity (Gödel 1949), numerous studies have shown that it is possible, in principle, to have local closed timelike curves with adequate local boundary conditions, essentially decoupled from the overall geometry and topology of the universe. This open the road to creating such curves by intentional means, the true "spacetime engineering". If we accept this possibility, it is acceptable that intelligent beings will, in the fullness of time, indeed perform such engineering feats; from a plethora of possibilities offered by closed timelike curves, hardly anything is more interesting than intervening in the early epochs of the universe to make it more conductive to high complexity and advanced cognition. Of course, everything related to the closed timelike curves is bound to be highly speculative, since the causal structure of spacetimes containing them is bound to be extremely complex and counterintuitive; for some of the stranger philosophical aspects of such spacetimes see Richmond $(2003,2004)$ and references therein.

This would be analogous to the original Omega-point theory of Pierre Teilhard de Chardin, just without its obvious theological baggage (de Chardin 1975). The idea here is that the Omega-point formation in distant future is not only a concrete future event which requires a particular state-ofaffairs, possible only with further advance of intelligence and technology. Far more than that: the emergence of an Omega-point analog means reaching maximal complexity of human cognition, joining with divine omniscience and standing outside of the perceived flow of time. This last point, stepping into eternity will, according to Teilhard's scenario, enable co-presence of the Omega-point in all previous moments of world's history, including the early epochs before the advent of human species, before the formation of Earth and indeed before any life or cognition was possible. In a cartoonish simplification, Teilhardian deity evolves in distant future in order to reach back into every moment of time, including those decisive for its own evolution.

A modernized version has been offered by Tipler (1994) within a framework of topologically closed, finite universe which satisfies precise boundary conditions. Tipler's hypothesis is philosophically and even theologically interesting (e.g., Birtel 1995) even if it can be considered falsified after the discovery of large dark energy in 1998. Simply speaking, recollapsing Friedman model required by Tipler is inconsistent with both the age and the physical composition of the universe in the new standard cosmology (for an introduction, see Liddle 2015). Philosophical interest for Tipler's

\footnotetext{
${ }^{13}$ Quotation marks are necessary here, since chronological ordering - and hence past, present and future does not exist, strictly speaking, in the presence of closed timelike curves. Each two points on such a curve are both in the "past" and in the "future" of one another, making these terms incoherent. However, the great interest of theoreticians in Gödel-like cosmological models led to a compromise understanding of "weak" chronology or nearly causal spacetime, insofar closed timelike curves are rare in spacetime (e.g., Monroe 2008).
} 
hypothesis is similar to the one for the steady-state cosmology of Bondi, Gold, and Hoyle from 1940s: it might help us clarify some of the most basic ideas of cosmology and metaphysics, likely to be found in many other places as well. ${ }^{14}$ On the other hand, instead of the Omega-point, we can today envision advanced technological singularity (e.g., Chalmers 2010), which anyway can represent an attractor in the space of all evolutionary trajectories of intelligent beings. In such a manner, it is possible at least in principle to remove supernaturalistic elements from the Teilhardian picture.

This model has also been prefigured by Wheeler, who understood, even in the early days of modern precision cosmology that the future of universes containing life and intelligence is essentially different from the future of universes devoid of such forms of complex organization of matter. Universal cosmic evolution proceeds, in a sense, through the emergence of complexity corresponding to intelligent observers and their civilizations. Even if we today can perceive only the smallest and most limited and local forms of the process, it is still no less real in the fullness of time (Wheeler 1988, p. 6):
Minuscule though the part is today that such acts of observer-participancy play in the scheme of things, there are billions of years to come. There are billions upon billions of living places yet to be inhabited. The coming explosion of life opens the door to an all-encompassing role for observer- participancy: to build, in time to come, no minor part of what we call its past-our past, present and future-but this whole vast world.

Obviously, most of discussions of place and long-term future of intelligent observers in the universe rely on some assumptions pertaining to the relevant motivations and capacities of intelligent communities. The present metaphysical theme, therefore, intertwines with both physical eschatology and SETI studies (as noted recently by several thinkers, notably Vidal [2010, 2014], Gardner [2007], and Stewart [2010]). This is a further instance of the "properly" scientific aspect of an age-old metaphysical reflection.

Basic common ingredient of these ideas is the "return into the past", joining our subjective past and future into the single entangled network of causes and consequences, from which habitability of our universe emerges. This habitability is manifested, of course, through the empirical fine tunings. Closed timelike curves are here mostly as a "vehicle" to achieve the desired aim; of course, the existence of such "aim" is a teleological element, but as Paul Davies correctly emphasizes, something which is "akin to teleology" cannot be avoided if we wish to have a real alternative to the multiverse hypothesis (Davies 2007). The complexity of any situation featuring closed timelike curves requires a kind of advanced information processing which will, hopefully, be achieved in human or posthuman future. ${ }^{15}$ Of course, even if humanity becomes extinct, another intelligent species based on the same physical laws of our universe could play the very same role. One should not forget that entirely

\footnotetext{
${ }^{14}$ Interestingly enough, Sir Fred Hoyle was in his later work, quite interested in the possibility of backward causation, as testified by the discussion in his Intelligent Universe (Hoyle 1983, esp. pp. 211-239). We are grateful to an anonymous referee for bringing our attention to this fascinating piece of history.

${ }^{15}$ Extreme complexity introduced by closed timelike curves even in previously quite simple everyday situations has been brilliantly demonstrated by the superb thriler of Shane Carruth Primer (Carruth 2004). This movie also highlights another possible way of falsifying the present hypothesis: if artificially created closed timelike curves cannot reach further into the past than the moment of their creation, it is impossible to use them for increasing habitability of the universe.
} 
legitimate, even conservative, explanatory hypotheses within philosophy of time occasionally postulate divergent information processing powers, at least in principle (e.g., Arsenijević 1986).

\section{Simulations and coevolution}

So, suppose that the challenge is accepted, and coevolution is accepted as a viable explanatory hypothesis (or a category of hypotheses), on a par with the design and the multiverse. What do we do next? How can we proceed to elaborate more on the hypothesis and offer specific predictions? In other words, how do we bridge over the gap separating metaphysics from physics in instrumental terms?

We suggest that the best long-term possibility lies with numerical simulations of coevolutionary models. In a manner similar to the experiments in artificial life (A-life), one could observe the increase in complexity of simulated entities (Ray 2003), but in the same time letting the digital environment be causally connected to the achieved levels of complexity of subsystems. In other words, not create a simulated ecosystem with somewhat fluctuating parameters describing local conditions in order to observe evolution within it, but create a family of ecosystems whose (global) rules themselves are subject to change; not random change, but change which would be somehow connected to what has happened historically in the simulation so far. In other words, by deploying large-scale numerical simulations of evolving systems, we study complexity arising, to use the wise formulation of Iris Fry, neither by chance nor by design (Fry 2000; see also contributions to the excellent anthology of Lineweaver, Davies, \& Ruse 2013). While there is some controversy over the epistemic status of numerical simulations in modern science (e.g., Parker 2009; Winsberg 2010), it is undoubted that our scientific understanding got enormously strengthened since they became widely used with the advent of the "informatic revolution". Some of the state-of-the-art simulations are already achieving unprecedented levels of complexity and are utilized exactly for astrobiological purposes (e.g., Vukotić et al. 2016); it is clear, however, that those are only very first, crude steps in what is a very broad and long road in front of us. Testing the co-evolutionary hypothesis by numerical simulations of evolution of complexity in particular - still necessarily simplified - context offers a previously unknown way of making justified inferences from simulation results to conclusions about metaphysical "target" systems. Very encouraging steps in this direction have recently been undertaken, in particular by the team of Paul C. W. Davies (Pavlic et al. 2014; Adams et al. 2016).

While there has been long-standing attempts (sometimes a bit tongue-in-cheek) to empirically test the Design and the Multiverse hypotheses (e.g., Hsu and Zee 2006), most researchers would probably conclude that these are empirically untestable in their strongest versions. Therefore, they have been regarded as traditional metaphysical concepts. Such a strong division might have become obsolete already, since virtual worlds could now be created and evolved, at least below a particular (quickly moving!) threshold of complexity. Application of numerical simulations in studying coevolution of the universe and observers would overcome one of the common philosophical criticisms raised against numerical experiments, as opposed to the classical experiments, namely that numerical experiments by their very design rarely test the basic theory in contrast to establishing credibility of hypotheses derived from such a basic theory (Winsberg 2010). Claims about fine tuning of the universe can hardly be construed as being derived from a particular physical theory; rather, all 
theories have to conform to the grounding implications of habitability. It follows that the usage of numerical experiments is less constrained in this metaphysico-cosmological narrative than it is in the conventional physical and cosmological applications.

\section{Discussion}

Explaining fine tunings through coevolution of the physical world and observers is the least studied explanatory option in at least three quarters of the century long history of this (meta)problem. Insofar we wish to seriously face this issue on the shifting boundary of physics and metaphysics, and especially if we wish to resist fruitless and corrosive influence of explanatory nihilism, we would do very good to take coevolution seriously and to build models in which its advantages could show. Two models sketched above represent only some of the options within the framework of the "third way" of coevolution (Davies 2007). Of course, from the discussion above it is clear that a philosophical maneuver could be performed in such a way that the endosymbiotic model is connected to the multiverse hypothesis, and the model with closed timelike curves is connected to the design hypothesis. Although it does not seem particularly interesting or productive, it still shows how small is the number of options at our disposal when dealing with the problem of such generality as the origin of fine tunings of the observable universe. This circumstance presents in itself another argument against explanatory nihilism: we need not take that road yet, since we can indeed eliminate all but a few contending theories.

Finally, as much as in economics or sports, the strength of a player in philosophy depends not just only on her intrinsic properties, but also on the strength of her rivals. The Design hypothesis is unpopular for the reasons permeating science since the epoch of Enlightenment: it is commonly felt that by abandoning naturalism we would lose much and gain nothing substantial in terms of insight. In addition, in the concrete case of fine tunings of the universe, the design hypothesis offers only a dilluted and weak form of explanation - since we have no insight into the mind or motivation of the Designer, we could state that the Designer may create fine-tuned universe, but hardly anything more than that. By the same token, the Designer may create the multiverse, or a mechanism of evolving universes (e.g., self-reproducing universes inside black holes; cf. Smolin 1997) - or the coevolution processes discussed above. Arbitrage between these options seems fruitless since the Designer's motivation is inaccessible. In the opposite corner of the ring, although the multiverse hypothesis received strong independent support from advances in cosmology and string theory, there are still many difficulties; for instance, it could be argued that models of the multiverse considered thus far are either too small or too big (Soler Gil \& Alfonseca 2013). It is not just the fact that it will be long before string theory (in the form of M-theory or any other version) is empirically tested, but also the fact of life is that wider explanatory basis usually provide with livelier research activity. This is especially true in the interdisciplinary contexts which are still fighting for their legitimacy, as is the case with this aspect of "practical metaphysics".

Both models of co-evolution considered here contain a dose of something which will undoubtedly be characterized as "mysticism", usually associated with pre-analytical or even pre-Kantian philosophical tradition. Alternatively, it could be subsumed into science-fictional speculation in the spirit of, say, 
Olaf Stapledon (especially see Stapledon 1937) or Karl Schroeder (2005). As the philosopher Robert Nozick best put, however: ${ }^{16}$

\begin{abstract}
More than clarifying the issues somewhat, I wish I could resolve the question of whether reality is as the mystic describes it. I take the question, and the mystics' experiences, very seriously, which some will think immediately is a great mistake. (But do they think this only because they already assume a background theory that discounts the mystics' experiences; if so, what led them to that theory?) For the purposes of philosophical explaining and understanding, we need not resolve the question< it suffices to consider, elaborate, and keep track of the hypotheses.
\end{abstract}

Today, this could be done, as argued above, by using numerical experiments and simulations. Considering the emergence of numerical experiments as the "third kingdom" between empirical and theoretical research, they seem most convenient for understanding counterfactual state-of-affairs like universes without fine tunings or with different fine tunings and different conditions for evolution of complexity. Although there are many quite involved details which need to be tracked and elaborated, in the spirit of Nozick's quote, it does not seem that there are insurmountable obstacles for a research programme testing models of coevolution in a correspondingly rich parameter space.

A takeaway for the general philosophy of science could be another important instance of what George Ellis calls top-down causation (Ellis et al. 2012; Ellis 2015) and what has become a rather hot topic in recent years (e.g., Okasha 2012). The importance of top-down causation as opposed - but complementary - to the conventional reductionist bottom-up has been recognized in more and more fields, but it is exactly the top-heavy evolutionary processes, like the Darwinian selection, which carry the greatest weight in the discussions. The obvious relevance could be found even by choice of examples; thus Ellis writes (Ellis 2015, pp. 38-39):

\title{
Top down effects clearly occur when lower level entities cannot exist outside their higher level context (again, a common effect in biology, where symbiosis is rife)... bottom up emergence by itself is strictly limited in terms of complexity it can give rise to. Emergence of genuine complexity is characterised by a reversal of information flow from bottom up to top down.
}

Feynman's dramatic idea of understanding the qualitative meaning of the equations of physics, in what is arguably one of the most philosophical passages in his opus cited at the beginning of this paper, obtains true significance - and ceases to sound so shocking to the orthodox flock - only in this context. The very existence of high-level entities, such as frogs, composers, or morality, provides a starting point for the constraining flow of information top-down in Ellis' parlance. Their relationship with low-level entities becomes less surprising, less outrageous, and ultimately more susceptible to scientific understanding than it has been under the one-sided reductionist paradigm.

In contemporary philosophy of physics one often encounters attempts to "reign in" or even condemn allegedly excessive speculation present in contemporary theoretical physics (e.g., Callender 2004; Mosterín 2005). Instead of such Quixotian orientation to fighting windmills, perhaps a better way would be just the opposite: to accept the recipe which showed to be successful time and again, namely to take our best theoretical ideas entirely seriously and to philosophically analyze their

\footnotetext{
${ }^{16}$ Nozick (1981), p. 164.
} 
ultimate consequences. This new form of practical metaphysics, which has some time ago fortunately stopped being regarded as oxymoronic, has rich and intriguing future prospects. ${ }^{17}$

\section{REFERENCE}

Adams, A. M., Zenil, H., Davies, P. C. W., and Walker, S. I. 2016, “Formal Definitions of Unbounded Evolution and Innovation Reveal Universal Mechanisms for Open-Ended Evolution in Dynamical Systems" (preprint http://lanl.arxiv.org/abs/1607.01750).

Arsenijević, M. 1986, Prostor, Vreme, Zenon (Filozofsko društvo Srbije, Beograd, in Serbian).

Barnes, L. A. 2012, "The Fine-Tuning of the Universe for Intelligent Life," Publications of the Astronomical Society of Australia 29, 529-564.

Barrow, J. D. \& Tipler, F. J. 1986, The Anthropic Cosmological Principle (Oxford University Press, New York).

Birtel, F. T. 1995, “Contributions of Tipler's Omega Point Theory," Zygon 30, 315-327.

Bostrom, N. 2002, Anthropic Bias: Observation Selection Effects in Science and Philosophy (Routledge, New York).

Callender, C. 2004, "Measures, Explanation and the Past: Should 'Special' Initial Conditions Be Explained?" British Journal for the Philosophy of Science 55, 195-217.

Carr, B. J. \& Rees, M. J. 1979, "The anthropic principle and the structure of the physical world," Nature 278, 605-612.

Carroll, S. M. 2006, “Is Our Universe Natural?” Nature 440, 1132-36.

Carruth, S. (Director) 2004, Primer (DVD, StudioCanal).

Chalmers, D. J. 2010, "The Singularity: A Philosophical Analysis," Journal of Consciousness Studies 17, 7-65.

Ćirković, M. M. 2012, The Astrobiological Landscape: Philosophical Foundations of the Study of Cosmic Life (Cambridge University Press, Cambridge).

Cushing, J. T. 1985, "Is there just one possible world? Contingency vs. the bootstrap," Stud. Hist. Phil. Sci. 16, 31-48.

\footnotetext{
${ }^{17}$ The authors wish to thank Dušan Pavlović for help in obtaining some of the literature, and Slobodan Perović, Vojin Rakić and Anders Sandberg on pleasant and useful discussions. Four anonymous referees for Foundations of Science offered helpful suggestions which immensely improved a previous version of this manuscript. MMĆ acknowledges financial support from the Ministry of Education, Science and Technological Development of the Republic of Serbia through the project \#ON176021.
} 
Davies, P. C. W. 2006, The Goldilocks Enigma: Why Is the Universe Just Right For Life? (Allen Lane, London).

Davies, P. C. W. 2007, “Universes galore: where will it all end?" in Universe or Multiverse?, ed. by Bernard Carr (Cambridge University Press, Cambridge), pp. 487-505.

de Chardin, P. T. 1975, The Phenomenon of Man (Harper \& Row, New York).

Ellis, G. F. R. 2015, "Recognising Top-Down Causation," in A. Aguirre et al. (eds.) Questioning the Foundations of Physics (Springer, ), 17-44.

Ellis, G. F. R., Noble, D., \& O'Connor, T. 2012, “Top-down causation: an integrating theme within and across the sciences?" Interface Focus 2, 1-3.

Frayn, M. 2006, The Human Touch: Our Part in the Creation of a Universe (Faber and Faber, London).

Fry, I. 2000, The Emergence of Life on Earth: A Historical and Scientific Overview (Rutgers University Press, New Brunswick).

Gardner, J. N. 2007, The Intelligent Universe: Al, ET, and the Emerging Mind of the Cosmos (New Page Books, Pompton Plains, NJ).

Gödel, K. 1949, „An Example of a New Type of Cosmological Solutions of Einstein's Field Equations of Gravitation," Reviews of Modern Physics 21, 447-450.

Gonzalez-Diaz, P. F. 2011, "Life originated during accelerated expansion in the multiverse," Physics Essays 24, 445-453.

Hinshaw, G. et al. 2013, "Nine-Year Wilkinson Microwave Anisotropy Probe (WMAP) Observations: Cosmological Parameter Results," The Astrophysical Journal Supplement Series 208, no. 2.

Hogan, C. J. 2000, "Why the Universe is Just So," Reviews of Modern Physics 72, 1149-61.

Hoyle, F. 1983, The Intelligent Universe (Michael Joseph Limited, London).

Hsu, S. and Zee, A. 2006, “Message in the sky," Modern Physics Letters A 21, 1495-1500.

Kutschera, U. \& Niklas, K. J. 2005, "Endosymbiosis, cell evolution, and speciation," Theory in Biosciences 124, 1-24.

Lineweaver, C. H., Davies, P. C. W. \& Ruse, M. (eds.) 2013, Complexity and the Arrow of Time (Cambridge University Press, Cambridge).

Manson, N. A. 2000, “There Is No Adequate Definition of 'Fine-tuned for Life,'” Inquiry 43, 341-352.

Maynard Smith, J. \& Szathmary, E. 1997, The Major Transitions in Evolution (Oxford University Press, Oxford).

McFadden, G. I. 2001, "Primary and secondary endosymbiosis and the origin of plastids," J Phycology 37, 951-959. 
McGrew, T., McGrew, L., \& Vestrup, E. 2001, "Probabilities and the Fine-Tuning Argument: a Sceptical View," Mind 110, 1027-1038.

Monroe, H. 2008, “Are Causality Violations Undesirable?” Foundations of Physics 38, 1065-1069.

Mosterín, J. 2005, “Anthropic Explanations in Cosmology," in Hajek, Valdés \& Westerstahl (eds.), Proceedings of the 12th International Congress of Logic, Methodology and Philosophy of Science (North-Holland Publishing, Amsterdam), 441-471.

Nozick, R. 1981, Philosophical Explanations (Harvard University Press, Cambridge).

Oberhummer, H., Csoto, A., and Schlattl, H. 2000, "Stellar Production Rates of Carbon and Its Abundance in the Universe," Science 289, 88-90.

Okasha, S. 2012, "Emergence, hierarchy and top-down causation in evolutionary biology," Interface Focus 2, 49-54.

Parker, W. 2009, "Does Matter Really Matter? Computer Simulations, Experiments and Materiality," Synthese 169, 483-496.

Pavlic, T. P., Adams, A. M., Davies, P. C. W., \& Walker, S. I. 2014, "Self-referencing cellular automata: A model of the evolution of information control in biological systems," in H. Sayama, J. Rieffel, S. Risi, R. Doursat, \& H. Lipson (eds.) Proceedings of the Fourteenth International Conference on the Synthesis and Simulation of Living Systems, pp. 522-529 (preprint http://lanl.arxiv.org/pdf/1405.4070).

Ray, T. S. 2003 "An Evolutionary Approach to Synthetic Biology: Zen in the Art of Creating Life," in A. Ghosh and S. Tsutsui, Advances in Evolutionary Computing (Springer, New York), 479-517.

Rescher, N. 2000, Process Philosophy: A Survey of Basic Issues (Univ. of Pittsburgh Press, Pittsburgh).

Richmond, A. 2003, “Recent Work: Time Travel," Philosophical Books 44, 297-309.

Richmond, A. 2004, “Gödelian Time-Travel and Anthropic Cosmology," Ratio 17 , 176-190.

Sagan, L. 1967, "On the origin of mitosing cells," Journal of Theoretical Biology 14, 255-274.

Schroeder, K. 2005, The Lady of Mazes (Tor Books, New York).

Smolin, L. 1997, The Life of the Cosmos (Oxford University Press, Oxford).

Soler Gil, F. J. \& Alfonseca, M. 2013, "Fine Tuning Explained? Multiverses and Cellular Automata," Journal for the General Philosophy of Science 44, 153-172.

Stapledon, O. 1937, Star Maker (Methuen, London).

Stapp, H. 2007, Mindful Universe: Quantum Mechanics and the Participating Observer (Springer Verlag, Berlin).

Stewart, J. E. 2010, "The Meaning of Life in a Developing Universe," Foundations of Science 15, 395409. 
Tegmark M. \& Rees, M. J. 1998, “Why is the Cosmic Microwave Background Fluctuation Level 10-5?" Astrophysical Journal 499, 526-32.

Thompson, J. N. 1994, The Coevolutionary Process (University of Chicago Press, Chicago).

Tipler, F. J. 1994, The Physics of Immortality (Doubleday, New York).

Vidal, C. 2010, "Computational and biological analogies for understanding fine-tuned parameters in physics," Foundations of Science 15, 375-393.

Vidal, C. 2014, The Beginning and the End: The Meaning of Life in a Cosmological Perspective (Springer, New York).

Vukotić, B., Steinhauser, D., Martinez-Aviles, G., Ćirković, M. M., Micic, M., and Schindler, S. 2016, "'Grandeur in this view of life': N-body simulation models of the Galactic habitable zone ," Monthly Notices of the Royal Astronomical Society 459, 3512-3524.

Wheeler, J. A. 1975, "The universe as home for man," in O. Gingerich (ed.) The Nature of Scientific Discovery (Smithsonian Institution Press, Washington), 261-296.

Wheeler, J. A. 1977, "Genesis and observership," in R. E. Butts and J. Hintikka, (ed.), Foundational Problems in the Special Sciences (Dordrecht: D. Reidel), pp. 3-33.

Wheeler, J. A. 1988, "World as system self-synthesized by quantum networking," IBM Journal of Research and Development 32, 4-15.

Windsor, H. H. (ed.) 1907, “Cart before the horse," Popular Mechanics April issue, 425.

Winsberg, E. 2010, Science in the Age of Computer Simulation (The University of Chicago Press, Chicago). 